\title{
Power spectra methods for a stochastic description of diffusion on deterministically growing domains
}

\author{
Thomas E. Woolley, ${ }^{1,}{ }^{*}$ Ruth E. Baker, ${ }^{1}$ Eamonn A. Gaffney, ${ }^{1}$ and Philip K. Maini ${ }^{1,2}$ \\ ${ }^{1}$ Centre for Mathematical Biology, Mathematical Institute, University of Oxford, Oxford, OX1 3LB, United Kingdom \\ ${ }^{2}$ Department of Biochemistry, Oxford Centre for Integrative Systems Biology, University of Oxford, Oxford, OX1 3QU, United Kingdom
}

(Received 6 April 2011; published 10 August 2011)

\begin{abstract}
A central challenge in developmental biology is understanding the creation of robust spatiotemporal heterogeneity. Generally, the mathematical treatments of biological systems have used continuum, mean-field hypotheses for their constituent parts, which ignores any sources of intrinsic stochastic effects. In this paper we consider a stochastic space-jump process as a description of diffusion, i.e., particles are able to undergo a random walk on a discretized domain. By developing analytical Fourier methods we are able to probe this probabilistic framework, which gives us insight into the patterning potential of diffusive systems. Further, an alternative description of domain growth is introduced, with which we are able to rigorously link the mean-field and stochastic descriptions. Finally, through combining these ideas, it is shown that such stochastic descriptions of diffusion on a deterministically growing domain are able to support the nucleation of states that are far removed from the deterministic mean-field steady state.
\end{abstract}

DOI: 10.1103/PhysRevE.84.021915

PACS number(s): 87.15.Vv, 82.20.Uv, 87.18.Tt, 02.50.Fz

\section{INTRODUCTION}

How spatial and temporal complexity emerges from near homogeneity is one of the fundamental questions of developmental biology that remains poorly understood, especially the question of how robustness is maintained despite the presence of numerous sources of noise. Thus, not only is producing plausible theoretical models of biological heterogeneity difficult, it is further complicated by the problem of generating robustness [1], i.e., once we can generate a pattern, how do we ensure that this pattern is consistently reproducible in the face of perturbation [2]?

Many mathematical models of biochemical systems have been proposed to account for the emergence of complexity $[3,4]$. In general, they have consisted of deterministic systems of differential equations, which do not consider the stochastic nature of chemical interactions. Hence, very few models have considered biological systems where the active populations have very low copy numbers, such as in the genome of the bacterium Escherichia coli, where around 1700 important proteins are present in small numbers. For example, there are only 10-30 molecules of the lac repressor, which is involved in the regulation of gene expression [5]. Thus, in such circumstances, the continuum hypothesis breaks down and we are forced to consider the potential stochastic effects of low copy numbers. Due to advances in computing power we are now able to return to these paradigm systems with the aim of modeling the primary biological phenomena while including stochastic effects, which, originally, would have made the simulations prohibitively slow.

Before we consider this connection between stochastic and deterministic dynamics, we must first clarify the type of noise we are trying to understand. Biologically, noise can be split into two different types, namely, intrinsic and extrinsic [6]. Although both are sources of stochasticity, their

*woolley@maths.ox.ac.uk mathematical treatments are very different [7]. Intrinsic noise is generated as an inherent part of the system and is due to random fluctuations in the population interactions [8]. Due to its intrinsic nature, the properties of internal noise need to be extracted from the imposed mathematical framework. External noise, in comparison, denotes fluctuations from any other source, e.g., domain effects or variations in temperature. Although the action of the external noise sources on the populations is unknown, a random variable can be applied to mimic their effects. Thus, in the case of external noise, since the random signal is explicitly added, the properties of the noise are chosen and controlled [9].

Much work has been done in analyzing and simulating the effects of external noise on reaction-diffusion pattern formation systems [9-14]. Simple forms of intrinsic noise have also been investigated $[6,8,15]$. However, until recently [16-19], there have been few extensions to the modeling of biological systems that have spatial or nonlinear temporal properties. Due to the inherent difficulties involved in analyzing stochastic systems, research has focused on efficient algorithms for simulation [20-26], thus producing statistics through ensemble data. However, there has been little analysis to complement these results $[27,28]$ and any advances in theory have frequently needed heuristic moment closure arguments in order to allow analytic treatment $[29,30]$.

Here we focus on diffusive systems, subject to intrinsic noise. Diffusion of the chemical constituents is modeled as a space-jump process [30]. The one-dimensional domain is partitioned into compartments of equal size and each compartment contains an indexed population. The individual particles of the chemical populations are then allowed to undergo unbiased random walks by jumping from one compartment to the next, i.e., each particle has an equal probability per unit time, or transition rate, of jumping to one of the neighboring boxes. We are interested in diffusion as it is a fundamental mechanism of transport that underpins a large number of biological processes [31-33]. We approach the biological system through a chemical master-equation formalism and 
use white-noise expansions to derive Fokker-Planck equations that allow us to characterize the properties of the noise [6]. This then brings us to one of the aims of the paper, which is to develop, clarify, and apply Fourier-transform techniques to the Fokker-Planck equation. These techniques give us insight into the potential spatiotemporal dynamics that are available in such systems. Although, by using these methods, we will be considering nonlinear complexity through linear analysis, it has been shown that linear dynamics can often afford a good approximation to the full nonlinear behavior, particularly near homogeneous steady states $[3,4,34]$.

Our next focus is to introduce an alternative treatment of growing domains. Growth has been hypothesized to play an important role in many areas of developmental biology, e.g., alligator tooth initiation [35,36] and fish pigmentation patterning [37]. Further, by coupling deterministic Turing reaction-diffusion patterning systems with growing domains, it has been shown that certain forms of growth robustly generate doubling wave mode sequences [38]. Thus it is with a view to considering robust pattern generation that we develop a framework that not only captures domain growth within stochastic systems but also enables analytical consideration of the weak-noise limit.

Heuristic arguments have been successful in linking the macroscopic and microscopic descriptions of growth using a box-splitting mechanism such that when the growth reaction occurs a compartment is chosen at random and replaced with two daughter compartments, each of which is the same size as the original compartment, with the original parent population split between them. However, this description of growth is unsuitable for considering the effects of noise on patterning mechanisms as this operation of box-splitting does not have a weak-noise limit (see Sec. II A). This can be demonstrated both analytically and intuitively: The process of splitting a population density in a single reaction is obviously a large perturbation; thus, increasing the active populations will produce an increase in noise, instead of a reduction. In order to bypass this problem of linking the microscopic and macroscopic scales we introduce a mesoscopic level of description through mapping the microscopic domain defined by Eulerian coordinates to a stationary Lagrangian domain, where, instead of manipulating the spatial variables, we simply alter the transition rates. The question of why we would want to do this is quite pertinent. This mapping allows us to not only link the mesoscopic and macroscopic scales without the need of moment closure heuristics but also to use the developed Fourier-transform theory in order to analyze complex spatial dynamics. Further, the stochastic realizations run much faster due to the coarser description of the domain, although by using the mesoscopic description we lose information on the microscopic scale. Finally, this Lagrangian description overcomes some of the flaws of the box-splitting growth approach. For instance, the time scale of growth is usually much smaller than the characteristic time scale of diffusion; thus the growth does not spontaneously affect only the splitting population, but rather the whole Lagrangian region of populations. The box-splitting method does have the advantage that the domain discretization will remain valid for all times as more spatial compartments are added as the domain grows. In contrast, due to the discretization being fixed in the Lagrangian description the domain will eventually become too coarse to resolve the spatial dynamics properly, at least for unbounded growth, and the well-mixed assumption of the Lagrangian compartment will break down. Thus we must fix an end time and ensure that the final size of the compartment is small enough to resolve any spatial dynamics. This refinement of the initial compartment size will always be possible, at least in principle.

Although we take a view that our active species are simple particle reagents in some biochemical system, where the intrinsic noise arises through random thermodynamic fluctuations of collisions, the developed methodology is much more general and can be applied to any biological system in which it is justified to assume that individuals act like interacting particles. The intrinsic noise is then generated through some other underlying process that causes the interactions to have a probability distribution rather than an exact rate. Further, if a particular population can be thought of as undergoing random movement, then diffusion may also be a good model for motility [39-41].

We start in Sec. II by introducing the stochastic framework in which we will work and extend this in Sec. III using the Fourier-transform techniques developed by McKane and Newman [16]. These methods deserve careful examination in order to clarify the role of complicating factors, such as boundary conditions, which can have a big effect on the analysis. Methods of modeling growth are discussed in Sec. IV and used in Sec. V to justify the mapping from the Eulerian domain, which is time-dependent, to the Lagrangian domain, which is time-independent. Comparisons of theory and simulation are then shown in Sec. VI, where we consider diffusion on a deterministically growing domain. This allows us to derive a scale parameter that indicates whether growth is able to cause consistent spatial heterogeneity. Finally, in Sec. VII we collect these results and interpret them with a view to understanding the effects of stochasticity and growth on diffusion.

\section{STOCHASTIC FRAMEWORK}

In this section we derive the basic methods that will allow analytical treatment of stochastic models [6,42]. However, before we are able to use such tools, we first need to introduce the chemical master equation (CME).

The CME of a reactive system is an exact description of the evolution of the probabilities of the active chemical species being in a certain state. By deriving the CME, the system is reduced to specifying only the populations and interactions between them while ignoring their specific positions and velocities. The justification for this lies in the conditions needed for the system to be considered well-mixed. Fundamentally, an assumption is made that the majority of molecular collisions are nonreactive. The net effect of these nonreactive collisions is to cause the positions of each particle to be uniformly random in the system volume. Thus, ignoring nonreactive collisions, only changes in populations due to defined reactions are considered [43].

Suppose in the system there are $K$ chemical populations $\left\{S_{1}, \ldots, S_{K}\right\}$ undergoing $J$ reactions $\left\{R_{1}, \ldots, R_{J}\right\}$. Since we will be primarily concerned with spatially extended systems 
the subscript $i=1, \ldots, K$ indexes over both the chemical population and its spatial location. Let $U_{i}(t)$ be the integer random variable of individuals of species $S_{i}$ and for simplicity define $\boldsymbol{U}(t)=\left[U_{1}(t), \ldots, U_{K}(t)\right]$ to be the state vector of the system at time $t$. Each reaction $R_{j}$ is specified by its stoichiometric vector $\boldsymbol{v}_{j}=\left(v_{1 j}, \ldots, v_{K j}\right)$ and the propensity function $a_{j}(\boldsymbol{u})$, where $\boldsymbol{u}$ is a single realization of $\boldsymbol{U}$ [44].

Definition 1.The stoichiometric vector $\boldsymbol{v}_{j}=\left(v_{1 j}, \ldots, v_{K j}\right)$ is the state change vector of reaction $R_{j}$. Thus, if the system is in state $\boldsymbol{u}$ and reaction $R_{j}$ occurs, then the state becomes $\boldsymbol{u}+\boldsymbol{v}_{j}[43]$.

Definition 2.The fundamental premise of stochastic chemical kinetics [43] is that the propensity function $a_{j}(\boldsymbol{u})$ is defined by

$$
a_{j}(\boldsymbol{u}) d t=\mathcal{P},
$$

where $\mathcal{P}$ is the probability, given $\mathbf{U}(t)=\boldsymbol{u}$, that one $R_{j}$ reaction will occur somewhere inside the system in the next infinitesimal time interval $[t, t+d t)$.

The stoichiometric matrix

$$
\boldsymbol{v}=\left\{v_{i j}\right\}_{i=1, \ldots, K}^{j=1, \ldots, J},
$$

and the propensity vector $\boldsymbol{a}^{T}=\left(a_{1}, \ldots, a_{J}\right)$ follow from these definitions. The exact form of the propensity function is proportional to the number of combinations of active particles available to undergo reaction $R_{j}$ [45]. The constant of proportionality, $\kappa_{j}$, which links the propensity function and the number of combinations of active particles, is known as the stochastic reaction rate. The rest of the theory follows from this and the Laws of Probability [46].

By defining $P(\boldsymbol{u}, t)$ as the probability of being in a state $\boldsymbol{u}$ at a time $t$ then the rate of change of $P$ over time is the net probability flux into the state $\boldsymbol{u}$, we obtain the general form of the CME,

$$
\begin{aligned}
\frac{\partial}{\partial t} P\left(\boldsymbol{u}, t \mid \boldsymbol{u}_{0}, t_{0}\right)= & \sum_{j=1}^{J}\left[P\left(\boldsymbol{u}-\boldsymbol{v}_{j}, t \mid \boldsymbol{u}_{0}, t_{0}\right) a_{j}\left(\boldsymbol{u}-\boldsymbol{v}_{j}\right)\right. \\
& \left.-P\left(\boldsymbol{u}, t \mid \boldsymbol{u}_{0}, t_{0}\right) a_{j}(\boldsymbol{u})\right] \\
\stackrel{\text { def }}{=} & \sum_{j=1}^{J}\left(R_{j}-1\right)\left[P\left(\boldsymbol{u}, t \mid \boldsymbol{u}_{0}, t_{0}\right) a_{j}(\boldsymbol{u})\right]
\end{aligned}
$$

Note that here we are using $R_{j}$ to denote the reaction path as well as the reaction operator. This abuse of notation should not cause confusion.

In the case of zeroth- and first-order reactions, i.e., reactions in which zero or one active species, respectively, reacts to form another, the CME is linear in $\boldsymbol{u}$ and can be solved analytically [47] or by using probability generating functions, which lead to first-order partial differential equations (PDEs) for the moments of the probability distribution [48]. Similarly, second-order reactions lead to second-order PDEs for the generating function. Hence, except for very special cases, second- and higher-order CMEs are not completely solvable analytically due to the nonlinearities in the system.

\section{A. Weak-noise expansion}

Although we may not be able to solve the CME exactly, except in certain special circumstances, we are able to systematically produce an approximation of it by expanding the equations in terms of a suitable system parameter, denoted $\Omega$, and assuming that, as this parameter is increased, the stochastic fluctuations become lower order. This allows separation of average, mean-field, and stochastic parts by yielding deterministic and probabilistic equations, respectively. By deriving equations for the mean-field dynamics and the effects of the noise about these, we are able to compare analytic results to ensemble data gained from stochastic simulations.

Motivated by the central limit theorem [46], we define a random variable $\eta_{i}$ (which is of order one as $\Omega \rightarrow \infty$ ) through the relation

$$
U_{i}=\phi_{i} \Omega+\eta_{i} \sqrt{\Omega} .
$$

In the case of multiple spatially extended populations we define $\Omega$ to be the order of magnitude of the smallest homogeneous steady state. Thus, by making $\Omega$ large, we ensure that all populations satisfy the weak-noise assumption. Here we consider only one spatially extended population and thus we fix $\Omega=U_{i}(0)=u(0)$ if the initial condition is homogeneous and $\Omega$ is the mean value of the $U_{i}(0), i=1, \ldots, K$, otherwise. Thus $\Omega$ does not have a spatial dependence. $\phi_{i}$ is the expected ratio of population at time $t$ to the initial population and is a dimensionless macroscopic variable. Since it is macroscopic we assume it is of order one compared to $\Omega$; thus the various orders of the model can be separated. The statistics of $U_{i}$ and $\eta_{i}$ are linked through the identification [6]

$$
P(\boldsymbol{U}, t)=\Pi(\eta, t) .
$$

Thus, in the limit,

$$
\lim _{\Omega \rightarrow \infty} \frac{\boldsymbol{U}}{\Omega}=\boldsymbol{\phi}=\left(\phi_{1}, \ldots, \phi_{K}\right) .
$$

This is consistent with the broadly accurate argument, identified by van Kampen [6], that the noise scales as square root of the population size. Also, it encapsulates the intuitive idea that, as the population grows, individual interactions become less important and we can treat the population as a continuum.

Using Taylor series, we are now able to expand the operator $R_{j} P(\boldsymbol{u}, t)$ about this function $\Pi$ and the microscopic propensity function $a_{j}\left(\boldsymbol{u}-\boldsymbol{v}_{j}\right)$ about the macroscopic rate $a_{j}(\phi)$ in terms of $\Omega$. Through evaluating these expansions and ignoring terms of order $1 / \sqrt{\Omega}$ and lower, we obtain from the CME

$$
\begin{aligned}
\frac{\partial \Pi}{\partial t} & -\sqrt{\Omega} \sum_{i=1}^{K} \frac{d \phi_{i}}{d t} \frac{\partial \Pi}{\partial \eta_{i}} \\
= & \sum_{j=1}^{J}\left(-\sqrt{\Omega} \sum_{i=1}^{K} v_{i j} \frac{\partial}{\partial \eta_{i}}+\frac{1}{2} \sum_{i=1}^{K} \sum_{l=1}^{K} v_{i j} v_{l j} \frac{\partial^{2}}{\partial \eta_{i} \partial \eta_{l}}\right) \\
& \times\left(\mathrm{a}_{j}(\boldsymbol{\phi})+\frac{1}{\sqrt{\Omega}} \sum_{i=1}^{K} \frac{\partial \mathrm{a}_{j}(\phi)}{\partial \phi_{i}} \eta_{i}\right) \Pi(\boldsymbol{\eta}) .
\end{aligned}
$$

Collecting terms of order $\sqrt{\Omega}$ leads to the macroscopic equation

$$
\sum_{i=1}^{K} \frac{d \phi_{i}}{d t} \frac{\partial \Pi}{\partial \eta_{i}}=\sum_{i=1}^{K} \sum_{j=1}^{J} \mathrm{a}_{j}(\boldsymbol{\phi}) v_{i j} \frac{\partial \Pi}{\partial \eta_{i}}
$$


which is satisfied by the definition of $\mathrm{a}_{j}(\boldsymbol{\phi})$. Collecting terms of order one gives rise to a Fokker-Planck equation [49], which defines the dynamics of the probability density

$$
\begin{aligned}
\frac{\partial \Pi}{\partial t} & =\sum_{j=1}^{J}\left(-\sum_{i, l=1}^{K} \frac{\partial \mathrm{a}_{j}}{\partial \phi_{l}} v_{i j} \frac{\partial\left(\eta_{i} \Pi\right)}{\partial \eta_{l}}+\frac{1}{2} \mathrm{a}_{j} \sum_{i, l=1}^{K} v_{i j} v_{l j} \frac{\partial^{2} \Pi}{\partial \eta_{i} \partial \eta_{l}}\right), \\
& =-\sum_{i, l=1}^{K} A_{i l} \frac{\partial\left(\eta_{l} \Pi\right)}{\partial \eta_{i}}+\frac{1}{2} \sum_{i, l=1}^{K} B_{i l} \frac{\partial^{2} \Pi}{\partial \eta_{i} \partial \eta_{l}},
\end{aligned}
$$

where [42]

$$
\begin{aligned}
& \boldsymbol{A}=\left\{A_{i l}\right\}=\left\{\partial[\boldsymbol{v a}]_{i} / \partial \phi_{l}\right\}, \\
& \boldsymbol{B}=\left\{B_{i l}\right\}=\boldsymbol{v} \operatorname{diag}(\mathrm{a}) \boldsymbol{v}^{T},
\end{aligned}
$$

and $\mathrm{a}^{T}=\left(\mathrm{a}_{1}, \ldots, \mathrm{a}_{J}\right)$.

\section{B. Spatially extended systems}

In order to proceed generally we must consider how the reactions affect the structure of the matrices $\boldsymbol{A}$ and $\boldsymbol{B}$. Since we are considering reaction-diffusion equations, it is important that we define diffusion on the microscopic and macroscopic scales. Although other descriptions are possible [24], here we model diffusion as a space-jump process [30] (see Fig. 1).

A domain of length $L$ is partitioned into $K$, onedimensional compartments of size $\Delta_{E}=L / K$ and each compartment contains an indexed population. Higher-dimensional domains can also be considered by replacing the onedimensional compartments with appropriate analogs [50]. For $K$ compartments in a one-dimensional domain with Neumann, zero-flux boundary conditions, each containing a species $S_{i}$, with population $U_{i}=\Omega \phi_{i}+\sqrt{\Omega} \eta_{i}$ and microscopic diffusion coefficient $d_{E}$, the reactions are

$$
S_{1} \underset{d_{E}}{\stackrel{d_{E}}{\rightleftharpoons}} S_{2} \underset{d_{E}}{\stackrel{d_{E}}{\rightleftharpoons}} \cdots \underset{d_{E}}{\stackrel{d_{E}}{\rightleftharpoons}} S_{K}
$$

Since these are first-order equations, the equations governing their mean values are equivalent to the deterministic reactions [48]

$$
\begin{aligned}
\frac{d \phi_{1}}{d t} & =d_{E}\left(\phi_{2}-\phi_{1}\right), \\
\frac{d \phi_{i}}{d t} & =d_{E}\left(\phi_{i-1}-2 \phi_{i}+\phi_{i+1}\right), \quad i=2, \ldots, K-1, \\
\frac{d \phi_{K}}{d t} & =d_{E}\left(\phi_{K-1}-\phi_{K}\right) .
\end{aligned}
$$

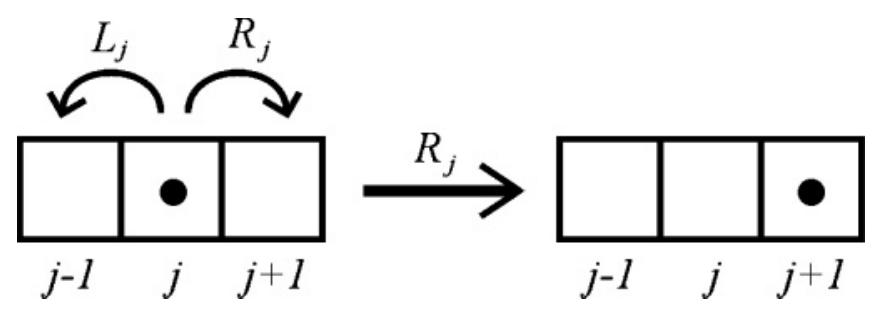

FIG. 1. Diagram of the space-jump description of diffusion. Each particle has equal probability per time unit of moving left as moving right. If, for example, the right diffusion reaction $R_{j}$ occurs, one particle from box $j$ moves to box $j+1$.
Comparing these equations with the second-order finitedifference approximation of the one-dimensional Laplacian with zero-flux boundary conditions, which is derived through Taylor expansions of the continuous formulation [51], shows that their descriptions are equivalent if $d_{E}=D / \Delta_{E}^{2}$, where $D$ is the macroscopic rate of diffusion [15]. Thus, because the reactions are linear, the expected value of the populations undergoing a stochastic description of diffusion approaches the deterministic diffusion equation as $\Delta_{E}$ tends to zero.

Similar to simulating a discretized reaction-diffusion PDE, if the discretized domain is too coarse the Eulerian boxes would be too large to justify a well-mixed assumption. Thus there is a restriction on the compartment size being too large. However, unlike PDEs, the compartment size may be restricted from being too small as the specific probability rate constants may depend on the size of the compartment; thus the limit $\Delta_{E} \rightarrow 0$ is not necessarily well defined [52]. Such problematic cases are not dealt with in the present context.

Using the matrix terminology developed in Sec. II A, the stoichiometric matrix $\boldsymbol{v}$ and the macroscopic transition rate a are given by

$$
\begin{gathered}
\boldsymbol{v}=\left(\begin{array}{cccccccccc}
-1 & 0 & \cdots & 0 & 0 & 0 & 1 & 0 & \cdots & 0 \\
1 & \ddots & \ddots & \vdots & \vdots & \vdots & -1 & \ddots & \ddots & \vdots \\
0 & \ddots & \ddots & 0 & \vdots & \vdots & 0 & \ddots & \ddots & 0 \\
\vdots & \ddots & \ddots & -1 & \vdots & \vdots & \vdots & \ddots & \ddots & 1 \\
0 & \cdots & 0 & 1 & 0 & 0 & 0 & \cdots & 0 & -1
\end{array}\right), \\
\quad a=\left(\begin{array}{c}
d_{E} \phi_{1} \\
\vdots \\
d_{E} \phi_{K} \\
d_{E} \phi_{1} \\
\vdots \\
d_{E} \phi_{K}
\end{array}\right) . \\
\end{gathered}
$$

$\boldsymbol{v}$ is a $K \times 2 K$ matrix. The first $K \times K$ elements are the population change vectors of diffusion to the right, e.g. the $j$ th column $(j<K)$ is the population transition of the reaction

$$
S_{j} \rightarrow S_{j+1}
$$

Similarly, for $j>K+1$, the $j$ th column is the population transition of

$$
S_{j} \rightarrow S_{j-1}
$$

The two zero columns in the center show that no particles may leave the domain, which corresponds to the Neumann boundary conditions. The $j$ th element of $\mathrm{a}$ is the corresponding weighting probability of the $j$ th reaction.

Using the definitions in Eqs. (10) and (11) we have

$$
\boldsymbol{A}=\left(\begin{array}{ccccc}
-d_{E} & d_{E} & 0 & \ldots & 0 \\
d_{E} & -2 d_{E} & d_{E} & \ddots & \vdots \\
0 & \ddots & \ddots & \ddots & 0 \\
\vdots & \ddots & d_{E} & -2 d_{E} & d_{E} \\
0 & \ldots & 0 & d_{E} & -d_{E}
\end{array}\right)
$$




$$
\boldsymbol{B}=\left(\begin{array}{ccccc}
2 d_{E} \phi & -2 d_{E} \phi & 0 & \ldots & 0 \\
-2 d_{E} \phi & 4 d_{E} \phi & -2 d_{E} \phi & \ddots & \vdots \\
0 & \ddots & \ddots & \ddots & 0 \\
\vdots & \ddots & -2 d \phi & 4 d_{E} \phi & -2 d_{E} \phi \\
0 & \ldots & 0 & -2 d_{E} \phi & 2 d_{E} \phi
\end{array}\right),
$$

where $\phi$ is the homogeneous steady-state solution of the system of Eqs. (13)-(15). The symmetric, tridiagonal forms of $\boldsymbol{A}$ and $\boldsymbol{B}$ are the key features used when deriving their spatiotemporal Fourier transforms. Any other reactions that are added into the one population system are assumed to act within each compartment, thus reagents in box $i$ can only react with other reagents in box $i$. This means that any additional reactions simply add extra terms to the diagonals of $\boldsymbol{A}$ and $\boldsymbol{B}$; thus we only need consider a symmetric, tridiagonal matrix for the following analysis to be a general solution of the Fokker-Planck equation.

\section{FOURIER TRANSFORMS}

The Fokker-Planck equation (9) can be converted to its corresponding Langevin equation, which has the general form $[42,53]$

$$
\frac{d \zeta}{d t}=A \zeta+\lambda(t)
$$

where $\zeta=\left(\eta_{1}, \ldots, \eta_{K}\right)$ and $\lambda=\left(\lambda_{1}, \ldots, \lambda_{K}\right)$. The $\lambda_{i}$ are specified by the covariances

$$
\left\langle\lambda_{i}(t) \lambda_{j}\left(t^{\prime}\right)\right\rangle=B_{i j} \delta\left(t-t^{\prime}\right)
$$

where $\langle x\rangle$ is the mean value of $x$ over a number of simulations. Note that, since the $\lambda_{i}$ are only uniquely defined up to their covariances, the Langevin equation is nonunique [53]. However, since we work with only the covariances, this is not a problem that will concern us.

Temporal and spatial Fourier-transform theory can be applied to the Langevin equation (21) in order to discern inhomogeneous behavior [16-19,54,55]. In this section we recapitulate this theory, paying particular attention to the form of expansion used and the effects of boundary conditions while extending the theory to non-steady-state kinetics.

\section{A. Temporal Fourier transform}

In future work we will be primarily interested in the spatial transform, as the biological patterns we study are normally stationary. Since the temporal transform has applications in systems with no stable steady state, e.g., limit cycles, it is covered for completeness [56]. Further, we extend current analytical results to nonstationary kinetics, highlighting the importance of carefully handling the boundary conditions.

As we will see later (Sec. IIIB), the spatial Fourier transform reduces the set of coupled ordinary differential equations (ODEs) in Eq. (21) to a single uncoupled ODE. Hence, in this section we consider only a single species $S$ with population $U$ and neglect diffusion.

\section{Stationary kinetics}

To illustrate the difference between stationary and nonstationary kinetics, we first consider the simple system of logistic growth

$$
S \underset{\beta / \Omega}{\stackrel{\alpha}{\rightleftharpoons}} 2 S
$$

Using the theory set out in Sec. II A, the deterministic equation describing the mean-field dynamics of $U$ is

$$
\dot{\phi}=\alpha \phi-\beta \phi^{2},
$$

with stable steady state $\phi_{s}=\alpha / \beta$. Further, we can easily find that $\boldsymbol{A}=A=\alpha-2 \beta \phi_{s}=-\alpha$ and $\boldsymbol{B}=B=\alpha \phi_{s}+\beta \phi_{s}^{2}=$ $2 \alpha^{2} / \beta$, which allows the calculation of the Langevin equation

$$
\dot{\eta}=-\alpha \eta+\lambda,
$$

where $\lambda$ is given by Eq. (22). At this point we would like to apply the discrete temporal Fourier transform [57]

$$
\tilde{f}(\omega)=\Delta_{t} \sum_{i=0}^{M-1} \exp \left(I \omega \Delta_{t} i\right) f\left(\Delta_{t} i\right),
$$

where $f$ is a function that has been sampled $M$ times over an interval $[0, T]$ such that $\Delta_{t}=T / M$ and $I$ is the imaginary unit.

The Fourier transform of $\eta$ is simply $\tilde{\eta}$ and when previously taking the temporal Fourier transform of the time derivative, the approximation $[16,54]$

$$
\tilde{\dot{\eta}}=-I \omega \tilde{\eta},
$$

has been used. However, this does not encompass any contribution from the initial and final values, as they are assumed to be small. Here we use this approximation successfully, which is justified later. In Sec. III A 2 the approximation fails when it is applied to kinetics that do not tend to a stable steady state.

Applying the discrete Fourier transform to Eq. (25) and using the approximation in Eq. (27), we obtain

$$
\begin{aligned}
-I \omega \tilde{\eta} & =-\alpha \tilde{\eta}+\tilde{\lambda}, \\
\Rightarrow \tilde{\eta} & =\frac{\tilde{\lambda}}{\alpha-\omega I} .
\end{aligned}
$$

Further,

$$
\begin{aligned}
\left\langle\tilde{\lambda}(\omega) \tilde{\lambda}\left(\omega^{\prime}\right)\right\rangle= & \Delta_{t}^{2} \sum_{i=0}^{M-1} \sum_{j=0}^{M-1} \exp \left(I \omega \Delta_{t} i\right) \\
& \times \exp \left(I \omega^{\prime} \Delta_{t} j\right)\left\langle\lambda\left(\Delta_{t} i\right), \lambda\left(\Delta_{t} j\right)\right\rangle, \\
= & \Delta_{t}^{2} \sum_{i=0}^{M-1} \sum_{j=0}^{M-1} \exp \left(I \omega \Delta_{t} i\right) \\
& \times \exp \left(I \omega^{\prime} \Delta_{t} j\right) B \delta\left(\Delta_{t}(i-j)\right), \\
= & \Delta_{t} B \sum_{i=0}^{M-1} \exp \left(I\left(\omega+\omega^{\prime}\right) \Delta_{t} i\right),
\end{aligned}
$$

implying

$$
\langle\tilde{\lambda}(\omega) \tilde{\lambda}(-\omega)\rangle=\Delta_{t} B M .
$$




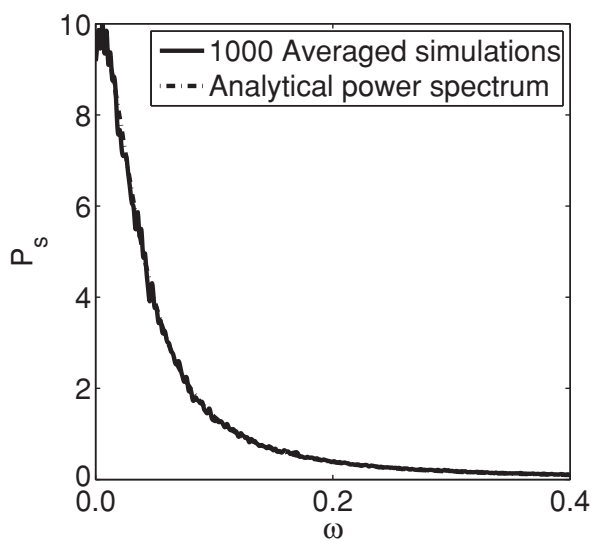

FIG. 2. Comparison of the power spectrum given by Eq. (32) (dashed line) and 1000 averaged replicates of the stochastic system (solid line), each scaled by a factor of $T$. The parameters are $\Omega=100$, $\alpha=0.1$, and $\beta=0.2$.

Thus we are able to calculate the power spectrum of the noise,

$$
P_{s}(\omega)=\frac{\left\langle|\tilde{\eta}|^{2}\right\rangle}{\Delta_{t} M}=\frac{2 \alpha^{2}}{\beta\left(\alpha^{2}+\omega^{2}\right)} .
$$

In Fig. 2 we compare the power spectrum Eq. (32) with the averaged ensemble of 1000 Fourier-transformed stochastic simulations, scaled by $\Delta_{t} M=T$. The figure clearly shows an extremely good fit between theory and simulation.

\section{Nonstationary kinetics}

Let us now consider kinetics that do not tend to a stationary steady state, e.g., exponential growth

$$
S \stackrel{r}{\rightarrow} 2 S .
$$

Proceeding as in Sec. III A 1 and using the approximation in Eq. (27), we derive the power spectrum to be

$$
P_{s}(\omega)=\frac{r}{r^{2}+\omega^{2}},
$$

where once again the spectrum has been scaled by a factor of $T$. Comparing this to scaled simulations in Fig. 3(a) shows that this time the comparison is not so good.

Similarly, if we consider linear growth

$$
\emptyset \stackrel{r}{\rightarrow} S,
$$

we obtain

$$
P_{S}(\omega)=\frac{\left\langle|\tilde{\eta}|^{2}\right\rangle}{T}=\frac{r}{\Omega \omega^{2}} .
$$

Qualitatively the curves in Fig. 3(b) are the same, but quantitatively the analytic solution is quite different. Thus, in order to obtain a better theoretical comparison to the ensemble power spectrum, we ignore the approximation in Eq. (27) and return to the Langevin equation.

\section{Corrected Fourier transform of the time derivative}

The Langevin equation arising from the weak-noise expansion method is always linear. Thus, following van Kampen [6], instead of taking the Fourier transform of the time derivative,

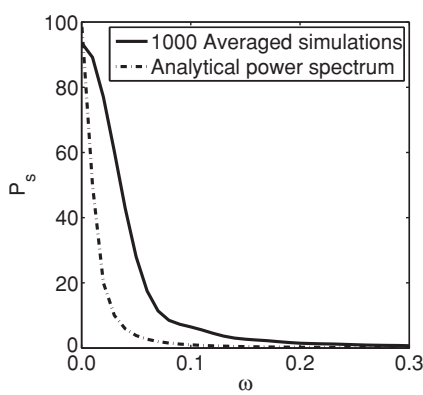

(a) $\Omega=1000, r=0.01$

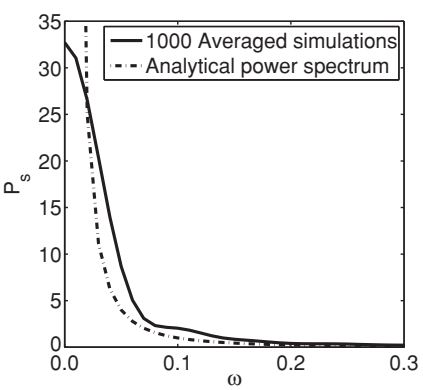

(b) $\Omega=1000, r=10$.
FIG. 3. Comparison of the power spectrum (dashed line) and 1000 averaged replicates of the stochastic system (solid line). Each result has been scaled by a factor of $T=100$. (a) Exponential growth power spectrum Eq. (34). (b) Linear growth power spectrum Eq. (36). The parameters are given beneath the figures.

we simply solve the linear ODE and take the Fourier transform of the resulting covariances.

Note in the following, general, derivation we consider the finite-time continuous transform as we expect the continuous and discrete transforms to correspond as $\Delta_{t} \rightarrow 0$ [57]. Let

$$
\dot{\eta}=c \eta+\lambda,
$$

where $c \neq 0$ is constant [58] and

$$
\left\langle\lambda\left(t^{\prime}\right) \lambda\left(t^{\prime \prime}\right)\right\rangle \stackrel{\text { def }}{=} g\left(t^{\prime}, t^{\prime \prime}\right) \delta\left(t^{\prime}-t^{\prime \prime}\right) .
$$

Since Eq. (37) is linear we can integrate the system to obtain

$$
\eta(t)=e^{c t} \int_{0}^{t} \lambda\left(t^{\prime}\right) e^{-c t} d t^{\prime},
$$

and thus

$$
\begin{aligned}
\left\langle\eta\left(t_{1}\right) \eta\left(t_{2}\right)\right\rangle & =e^{c\left(t_{1}+t_{2}\right)} \int_{0}^{t_{1}} \int_{0}^{t_{2}} g\left(t^{\prime}, t^{\prime \prime}\right) e^{-c\left(t^{\prime}+t^{\prime \prime}\right)} \delta\left(t^{\prime}-t^{\prime \prime}\right) d t^{\prime} d t^{\prime \prime}, \\
& =e^{c\left(t_{1}+t_{2}\right)} \int_{0}^{\min \left(t_{1}, t_{2}\right)} g\left(t^{\prime}, t^{\prime}\right) e^{-2 c t^{\prime}} d t^{\prime} .
\end{aligned}
$$

Simplifying the notation of $g\left(t^{\prime}, t^{\prime}\right)$ to $g\left(t^{\prime}\right)$ and applying the temporal transform gives

$$
\begin{aligned}
& \left\langle|\tilde{\eta}(\omega)|^{2}\right\rangle \\
& =\int_{0}^{T} \int_{0}^{T} e^{I \omega\left(t_{1}-t_{2}\right)} e^{c\left(t_{1}+t_{2}\right)} \int_{0}^{\min \left(t_{1}, t_{2}\right)} g\left(t^{\prime}\right) e^{-2 c t^{\prime}} d t^{\prime} d t_{1} d t_{2}, \\
& =\int_{0}^{T} \int_{0}^{t_{2}} e^{I \omega\left(t_{1}-t_{2}\right)} e^{c\left(t_{1}+t_{2}\right)} \int_{0}^{t_{1}} g\left(t^{\prime}\right) e^{-2 c t^{\prime}} d t^{\prime} d t_{1} d t_{2} \\
& \quad+\int_{0}^{T} \int_{t_{2}}^{T} e^{I \omega\left(t_{1}-t_{2}\right)} e^{c\left(t_{1}+t_{2}\right)} \int_{0}^{t_{2}} g\left(t^{\prime}\right) e^{-2 c t^{\prime}} d t^{\prime} d t_{1} d t_{2} \\
& =2 \int_{0}^{T} \int_{0}^{t_{2}} \cos \left[\omega\left(t_{1}-t_{2}\right)\right] e^{c\left(t_{1}+t_{2}\right)} \\
& \quad \times \int_{0}^{t_{1}} g\left(t^{\prime}\right) e^{-2 c t^{\prime}} d t^{\prime} d t_{1} d t_{2} .
\end{aligned}
$$

To make further progress we assume a form for $g$. If the kinetics have a stable steady state then $g$ will be a constant,

$$
g\left(t^{\prime}\right)=g,
$$


whence

$$
\begin{aligned}
\left\langle|\tilde{\eta}(\omega)|^{2}\right\rangle= & \frac{g\left(c^{3}+c \omega^{2}\right) T}{\left(c^{2}+\omega^{2}\right)^{2} c} \\
& -\frac{g\left[4 c^{2} \cos (\omega T)+4 \omega \sin (\omega T) c\right] e^{c T}}{2\left(c^{2}+\omega^{2}\right)^{2} c} \\
& +\frac{g\left(c^{2}+\omega^{2}\right) e^{2 c T}}{2\left(c^{2}+\omega^{2}\right)^{2} c}-\frac{g\left(-3 c^{2}+\omega^{2}\right)}{2\left(c^{2}+\omega^{2}\right)^{2} c} .
\end{aligned}
$$

Further, from the derivation of $\boldsymbol{A}$ in Sec. II A, $c$ will be the eigenvalue of the homogeneous system; thus, since we are assuming stable kinetics, $c$ will be negative and the exponentials will decay. Considering only the long-time solution leads to

$$
\left\langle|\tilde{\eta}(\omega)|^{2}\right\rangle=\frac{g T}{\left(\omega^{2}+c^{2}\right)} .
$$

Letting $c=\alpha$ and $g=2 \alpha^{2} / \beta$, we can compare this with the power spectrum of the logistic kinetics derived in Sec. III A 1. We see that additional terms associated with the temporal boundary values are of lower order; thus in the long-time limit they can legitimately be ignored.

Now suppose the kinetics are not stationary and thus $c>0$. This will then give a time-dependent $g=g\left(t^{\prime}\right)$. In general, Eq. (41) no longer has a closed-form solution; thus we must consider solutions on a case by case basis.

Returning to exponential kinetics, we see that $c=r$ and $g(t)=r \exp (r t)$. Thus

$$
\left\langle|\tilde{\eta}(\omega)|^{2}\right\rangle=\frac{-2 r e^{r T} \sin (\omega T)+\omega\left(e^{2 r T}-1\right)}{\omega\left(\omega^{2}+r^{2}\right)} .
$$

Figure 4(a) convincingly shows that Eq. (45) is a much better approximation than that originally derived in Eq. (34). However, the power spectrum of exponential growth does not tend to a limit when scaled by $1 / T$ and so the power spectrum is dependent on the final time value of the observed domain.

Finally, for the singular case of linear growth, the same procedure can be followed from Eq. (37), but this time $c=0$. This leads to

$$
\left\langle|\tilde{\eta}(\omega)|^{2}\right\rangle=2 \frac{r T}{\omega^{2} \Omega}-2 \frac{r \sin (\omega T)}{\omega^{3} \Omega} .
$$

Comparing this with the originally derived equation (36), we find a factor of 2 that was originally missing, as well a term of the form $r \sin (\omega T)$. From Fig. 4(b) we once again see excellent correspondence between theory and simulation.

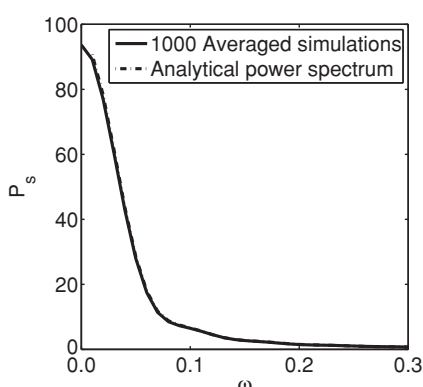

(a)

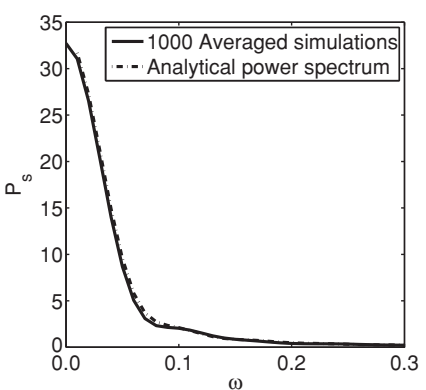

(b)
FIG. 4. Comparison of the corrected power spectrum (dashed line) and 1000 averaged replicates of the stochastic system (solid line). Each result has been scaled by a factor of $T=100$. (a) Exponential growth. Analytic power spectrum given by Eq. (45). (b) Linear growth. Analytic power spectrum given by Eq. (46). The parameters are the same as in Fig. 3.

\section{B. Spatial Fourier transform}

We now consider the discrete spatial Fourier transform of a spatially extended system with a population diffusing over the domain (see Sec. II B). In previous work, the utilized transform has been analogous to the temporal transform [18,54],

$$
\widehat{f}(k)=\Delta_{x} \sum_{j=1}^{K} \exp \left[-I k \Delta_{x}(j-1)\right] f\left(x_{j}\right),
$$

where $f$ is a function defined over a space that has been discretized into $K$ uniformly spaced points with spacing $\Delta_{x}$ such that $x_{j}=j \Delta_{x}$.

Since our simulations employ Neumann boundary conditions, which do not disappear on using the above transform, we choose instead to use the discrete Fourier cosine expansion [57]

$$
\widehat{f}(k)=\Delta_{x} \sum_{j=1}^{K} \cos \left[k \Delta_{x}(j-1)\right] f\left(x_{j}\right) .
$$

Note that the correction $(j-1)$ in the cosine function is simply to account for the fact that, spatially, we have defined our populations to start with an index one instead of zero. In contrast, the temporal initial condition is defined to occur at time $t=0$; thus the index for the temporal transformation starts at zero.

Taking the discrete spatial transform of the discrete Laplacian of $\eta$, with $\Delta_{x}=\Delta_{E}$, gives us

$$
\begin{aligned}
\Delta_{E} & \cos \left(k \Delta_{E} 0\right) d_{E}\left(-\eta_{1}+\eta_{2}\right)+\Delta_{E} \cos \left[k \Delta_{E}(K-1)\right] d_{E}\left(\eta_{K-1}-\eta_{K}\right)+\Delta_{E} \sum_{j=2}^{K-1} \cos \left[k \Delta_{E}(j-1)\right] d_{E}\left(\eta_{j-1}-2 \eta_{j}+\eta_{j+1}\right) \\
= & -2 d_{E} \widehat{\eta}_{k}+\Delta_{E} \cos \left(k \Delta_{E} 0\right) d_{E} \eta_{1}+\Delta_{E} \cos \left[k \Delta_{E}(K-1)\right] d_{E} \eta_{K}+\Delta_{E} \sum_{j=1}^{K-1} \cos \left(k \Delta_{E} j\right) d_{E} \eta_{j}+\Delta_{E} \sum_{j=2}^{K} \cos \left[k \Delta_{E}(j-2)\right] d_{E} \eta_{j}, \\
= & -2 d_{E} \widehat{\eta}_{k}+\Delta_{E} \cos \left(k \Delta_{E} 0\right) d_{E} \eta_{1}+\Delta_{E} \cos \left[k \Delta_{E}(K-1)\right] d_{E} \eta_{K} \\
& +\Delta_{E} \sum_{j=1}^{K-1}\left\{\cos \left[k \Delta_{E}(j-1)\right] \cos \left(k \Delta_{E}\right)-\sin \left[k \Delta_{E}(j-1)\right] \sin \left(k \Delta_{E}\right)\right\} d_{E} \eta_{j}
\end{aligned}
$$




$$
\begin{aligned}
& +\Delta_{E} \sum_{j=2}^{K}\left\{\cos \left[k \Delta_{E}(j-1)\right] \cos \left(k \Delta_{E}\right)+\sin \left[k \Delta_{E}(j-1)\right] \sin \left(k \Delta_{E}\right)\right\} d_{E} \eta_{j}, \\
= & 2 d_{E} \widehat{\eta}_{k}\left[\cos \left(k \Delta_{E}\right)-1\right]-k \Delta_{E}^{2} \frac{\cos \left(k \Delta_{E} 1\right)-\cos \left(k \Delta_{E} 0\right)}{k \Delta_{E}} d_{E} \eta_{1}+k \Delta_{E}^{2} \frac{\cos \left[k \Delta_{E}(K-1)\right]-\cos \left(k \Delta_{E} K\right)}{-k \Delta_{E}} d_{E} \eta_{K} .
\end{aligned}
$$

Taking the limit $\Delta_{E} \rightarrow 0+$ while keeping $\Delta_{E} K=L$ and $k$ fixed gives

$$
\begin{array}{r}
\lim _{\Delta_{E} \rightarrow 0+} \frac{\cos \left(k \Delta_{E} 1\right)-\cos \left(k \Delta_{E} 0\right)}{k \Delta_{E}}=\sin (0)=0, \\
\lim _{\Delta_{E} \rightarrow 0+} \frac{\cos \left[k \Delta_{E}(K-1)\right]-\cos \left(k \Delta_{E} K\right)}{-k \Delta_{E}}=\sin (k L) .
\end{array}
$$

Hence, by choosing $k=m \pi / L, m=0,1, \ldots, K-1$, we can ignore the boundary terms in Eq. (49) as they are of a lower order than the leading-order term $2 d_{E} \widehat{\eta}_{k}\left[\cos \left(k \Delta_{E}\right)-1\right]$. Further, by expanding the cosine function and remembering that, as $\Delta_{E} \rightarrow 0, d_{E} \Delta_{E}^{2}=D$ is finite,

$$
\lim _{\Delta_{E} \rightarrow 0} 2 d_{E} \widehat{\eta}_{k}\left[\cos \left(k \Delta_{E}\right)-1\right]=-k^{2} D \widehat{\eta}_{k},
$$

which is exactly the solution given by the continuous transform [18].

Thus the spatial Fourier cosine transform of the Langevin equation, generated by the space-jump description of diffusion [Eq. (21), where $\boldsymbol{A}$ and $\boldsymbol{B}$ are given by Eq. (20)], is given by

$$
\frac{d \widehat{\eta}_{k}}{d t}=2\left[\cos \left(k \Delta_{E}\right)-1\right] d_{E} \widehat{\eta}_{k}+\widehat{\lambda}_{k}
$$

Notice that the spatial cosine transform has reduced a set of $K$ coupled ODEs in Eq. (21) to a single ODE, Eq. (53), which can be solved for all $k=m \pi / L, m=0, \ldots, K-1$.

The explicit form of $\left\langle\widehat{\lambda}_{k}(t) \widehat{\lambda}_{k^{\prime}}\left(t^{\prime}\right)\right\rangle$ can be found similarly. Since $\boldsymbol{B}$ is tridiagonal and symmetric we can completely specify its elements by using Kronecker delta functions,

$$
B_{i j}=b_{0} \delta_{i, j}+b_{1} \delta_{|i-j|, 1},
$$

where the $b_{0}$ are the diagonal elements and the $b_{1}$ are the elements on the superdiagonals and subdiagonals. Once again we use a discrete cosine transform, which, as shown above, automatically incorporates the boundary conditions; thus

$$
\begin{aligned}
\left\langle\widehat{\lambda}_{k}(t) \widehat{\lambda}_{k^{\prime}}\left(t^{\prime}\right)\right\rangle= & \Delta_{E}^{2} \sum_{i, j=1}^{K} \cos \left[k \Delta_{E}(i-1)\right] \cos \left[k^{\prime} \Delta_{E}(j-1)\right] \\
& \times\left(b_{0} \delta_{i, j}+b_{1} \delta_{|i-j|, 1}\right) \delta\left(t-t^{\prime}\right)
\end{aligned}
$$

can be then simplified to

$$
\left\langle\widehat{\lambda}_{k}(t) \widehat{\lambda}_{k}\left(t^{\prime}\right)\right\rangle=\frac{\Delta_{E}^{2} K}{2}\left[b_{0}+2 \cos \left(k \Delta_{E}\right) b_{1}\right] \delta\left(t-t^{\prime}\right) .
$$

We have been able to apply the spatial Fourier cosine transform analytically due to the spatial homogeneity of the coefficients. If the coefficients were also constant in time, the temporal transform would cause no trouble and this case has been dealt with successfully [16,19,54,56,59,60]. For completeness, and as an example of using both transforms together, in the following section we apply both techniques to our stochastic description of diffusion.

\section{Temporal and spatial Fourier cosine transforms of diffusion}

In the preceding section we derived the spatial Fourier cosine transform of the space-jump description of diffusion. Thus, in order to use both transforms, we apply the temporal Fourier transform to Eq. (53). Although the system tends to a stationary steady state, meaning we could use approximation equation (27), we derive the equation fully using the same method as in Sec. III A 3.

If we let $C(k)=2 d_{E}\left[1-\cos \left(k \Delta_{E}\right)\right]$, then

$$
\widehat{\eta}_{k}=\exp [-C(k) t] \int_{0}^{t} \widehat{\lambda}_{k} \exp \left[C(k) t^{\prime}\right] d t^{\prime}
$$

This implies

$$
\left\langle\widehat{\eta}_{k}\left(t_{1}\right) \widehat{\eta}_{k}\left(t_{2}\right)\right\rangle=e^{-C(k)\left(t_{1}+t_{2}\right)} \int_{0}^{t_{1}} \int_{0}^{t_{2}}\left\langle\widehat{\lambda}_{k} \widehat{\lambda}_{k}\right\rangle e^{C(k)\left(t_{1}^{\prime}+t_{2}^{\prime}\right)} d t_{1}^{\prime} d t_{2}^{\prime}
$$

By considering the matrices in Eq. (20) and substituting the correct values into Eq. (56), we obtain

$$
\begin{aligned}
\left\langle\widehat{\lambda}_{k}(t) \widehat{\lambda}_{k}\left(t^{\prime}\right)\right\rangle & =2 \Delta_{E}^{2} K d_{E} \phi\left[1-\cos \left(k \Delta_{E}\right)\right] \delta\left(t-t^{\prime}\right), \\
& =\Delta_{E}^{2} K \phi C(k) \delta\left(t-t^{\prime}\right) .
\end{aligned}
$$

Thus

$$
\left\langle\widehat{\eta}_{k}(t) \widehat{\eta}_{k}(t)\right\rangle=\frac{\Delta_{E}^{2} K \phi}{2}\left(1-e^{-2 C(k) t}\right)
$$

Since $C(k) \geqslant 0$ the long-time covariance is time-independent,

$$
\lim _{t \rightarrow \infty}\left\langle\widehat{\eta}_{k}(t) \widehat{\eta}_{k}(t)\right\rangle=\left\{\begin{array}{lll}
0 & \text { if } & k=0 \\
\frac{\Delta_{E}^{2} K \phi}{2} & \text { if } & k \neq 0 .
\end{array}\right.
$$

As this is constant for all spatial frequencies, $k \neq 0$, then, as we would expect, the noise tends not to favor any one frequency over another. Thus stochastic effects in the diffusion equation do not produce a patterned state.

The temporal Fourier transform is derived by considering

$$
\begin{aligned}
& \left\langle\tilde{\widehat{\eta}}_{k}(\omega) \tilde{\widehat{\eta}}_{k}(-\omega)\right\rangle \\
& \quad=\int_{0}^{T} \int_{0}^{T} \exp \left[I \omega\left(t_{1}-t_{2}\right)\right]\left\langle\widehat{\eta}_{k}\left(t_{1}\right) \widehat{\eta}_{k}\left(t_{2}\right)\right\rangle d t_{1} d t_{2} .
\end{aligned}
$$




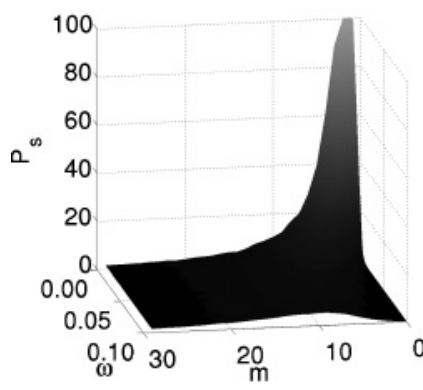

(a)

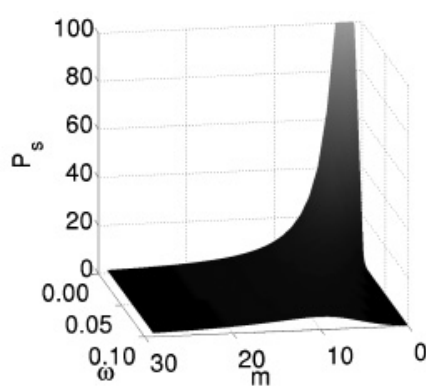

(b)

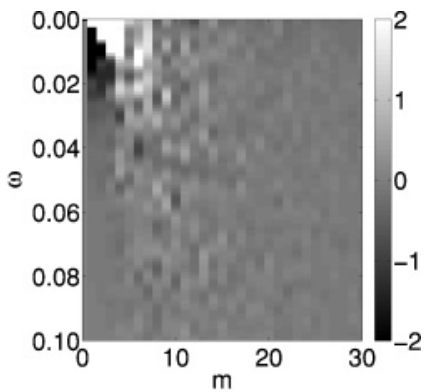

(c)

FIG. 5. Left: power spectra of 1000 averaged simulations. Middle: analytically derived power spectra; Eq. (64) scaled by $\Delta_{E} L T$. Right: analytical value minus the ensemble values. Note that $(k, \omega)=(0,0)$ is a singular point of Eq. (64); thus the analytic solution tends to infinity, whereas averaged simulations will always be finite. This is why the difference between the two spectra is close to zero everywhere, except near the origin. Note that, in order to see the variability around zero, we have fixed the color axis to be in the interval [ $-2,2]$. Thus, although the difference does become infinite near the origin, any value above 2 is colored white. The parameters used are $\phi=1, d_{E}=1, \Delta_{E}=1 / 100$, $T=1000$, and $L=1$.

The system is completely integrable and so, by suppressing the argument $k$ in $C(k)$, we obtain

$$
\begin{aligned}
\left\langle\left|\tilde{\hat{\eta}}_{k}(\omega)\right|^{2}\right\rangle= & \frac{\Delta_{E}^{2} K \phi}{2\left(\omega^{2}+C^{2}\right)^{2}}\left\{-\left(\omega^{2}+C^{2}\right)\right. \\
& \times[\cosh (2 C T)-\sinh (2 C T)] \\
& +\left(-4 \omega C \sin (\omega T)+4 C^{2} \cos [\omega T)\right] \\
& \times[\cosh (C T)-\sinh (C T)] \\
& \left.\times \omega^{2}-3 C^{2}+2 C T \omega^{2}+2 C^{3} T\right\} .
\end{aligned}
$$

The long-time form simplifies to

$$
\begin{aligned}
\left\langle\left|\tilde{\hat{\eta}}_{k}(\omega)\right|^{2}\right\rangle & =\frac{C \Delta_{E}^{2} K \phi T}{\left(\omega^{2}+C^{2}\right)}, \\
& =\frac{2 d_{E}\left[1-\cos \left(k \Delta_{E}\right)\right] \Delta_{E} \phi L T}{\omega^{2}+4 d_{E}\left[1-\cos \left(k \Delta_{E}\right)\right]^{2}},
\end{aligned}
$$

where $T$ is the total time of simulation and $L=K \Delta_{E}$ is the length of domain. Figure 5 shows that simulation once again compares favorably with theory. Note that $(k, \omega)=(0,0)$ is a singular point of Eq. (64) and as such the analytic solution tends to infinity near this point. Since the white-noise process is uncorrelated in time and space this is the expected behavior of the spectrum. However, the average of a finite number of discrete simulations is necessarily finite [56] and so we would expect that the approximation of the ensemble solution to the analytical solution to become worse near this point. This is clearly seen in Fig. 5(c).

\section{GROWTH}

As discussed in Sec. I, growth is of fundamental importance in the early stages of development. As such, there has recently been interest in deriving a stochastic description of growth in the space-jump framework [30] that is consistent with continuum theory [38].

Growth has previously been defined through a box-splitting operation on the populations [30]. We denote this an Eulerian form of growth. Initially, if an interval $[0, L]$ is split into $K$ boxes of length $L / K$ and the growth reaction occurs in the $j$ th box, a new box of size $L / K$ is created next to the $j$ th box, as in Fig. 6, and the populations are updated according to the rules

$$
\begin{aligned}
U_{K} & \mapsto U_{K+1}, \\
U_{K-1} & \mapsto U_{K}, \\
& \vdots \\
U_{j+1} & \mapsto U_{j+2}, \\
U_{j} & \mapsto\lceil U / 2\rceil_{j}+\lfloor U / 2\rfloor_{j+1},
\end{aligned}
$$

where $\lfloor x\rfloor$ is the largest integer less than $x$ and $\lceil x\rceil$ is the smallest integer greater than $x$. Note that various other splitting rules have been used, including splitting the population uniformly randomly over the two boxes; however, these have been seen to have little effect on the simulations (details not shown).

However, although microscopically this is a description of growth, the resulting CME that defines the operation does not have a weak-noise limit. Because of this, we use representations analogous to those of Crampin et al. [38] and map the growing Eulerian domain to a stationary Lagrangian domain where a weak-noise limit can be prescribed.

Let $x_{E}$ be the time-dependent Eulerian coordinate on a uniformly expanding, one-dimensional domain $[0, L(t)]$. The mean-field diffusion equation then takes the form $[38,61,62]$

$$
\frac{\partial \phi}{\partial t}+\frac{\partial}{\partial x_{E}}\left[v\left(x_{E}, t\right) \phi\right]=D \frac{\partial^{2} \phi}{\partial x_{E}^{2}} \quad \text { on } \quad[0, L(t)],
$$

where $v\left(x_{E}, t\right)$ is the flow generated by the growth. Since growth is uniform we assume that there exists a growth
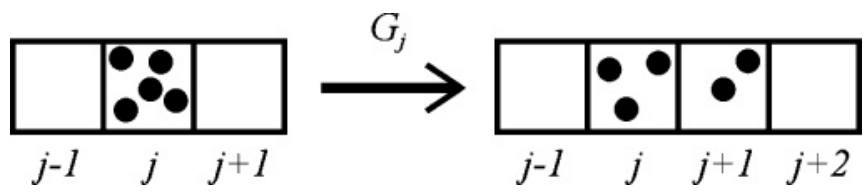

FIG. 6. Diagram illustrating domain growth. If the $j$ th box divides then a new box is created next to the $j$ th box. The population in the $j$ th box is split as evenly as possible between the $j$ th and $j+1$ st boxes and the rest of the populations are updated accordingly. 
function $n(t)$ such that $L(t)=L_{0} n(t)$ and $n(0)=1$. Thus we can relate the Eulerian coordinates to a time-independent Lagrangian coordinate $x_{L}$ through $x_{E}=x_{L} n(t)$. This then determines the local flow through

$$
v\left(x_{E}, t\right)=\frac{\partial x_{E}}{\partial t} .
$$

Converting to Lagrangian coordinates and dropping the subscript $L$,

$$
\frac{\partial \phi}{\partial t}+\frac{\dot{n}}{n} \phi=\frac{D}{n^{2}} \frac{\partial^{2} \phi}{\partial x^{2}} \quad \text { on } \quad\left[0, L_{0}\right] .
$$

Finally, by rescaling $\phi_{L}=\phi n$ and again dropping the subscript $L$, we remove the dilution terms to obtain

$$
\frac{\partial \phi}{\partial t}=\frac{D}{n^{2}} \frac{\partial^{2} \phi}{\partial x^{2}} \quad \text { on } \quad\left[0, L_{0}\right] .
$$

Thus, in the mean-field, we simply reproduce the diffusion equation with a time-dependent diffusion coefficient $D / n(t)^{2}$. We use this knowledge of the deterministic system to inform the transition rates, or the rate of jumping between the discretized compartments.

\section{LAGRANGIAN COORDINATES}

So far we have been able to formulate expressions for the power spectra of stochastically diffusing populations using both spatial and temporal transforms. However, with the introduction of domain growth, the diffusion coefficient gains a time dependence, as shown in Sec. IV. This dependence does not take a simple form akin to those studied in Sec. III A 2 and thus temporal Fourier transforms would have to be calculated numerically. However, since we are primarily considering stationary structures as models of inhomogeneous patterning, we concentrate purely on spatial Fourier-transform methods.

In order to model a time-dependent Eulerian domain we construct a fixed Lagrangian domain $[0, L]$ split into $K$ compartments of length $\Delta_{L}=L / K$ (see Fig. 7). Compartment

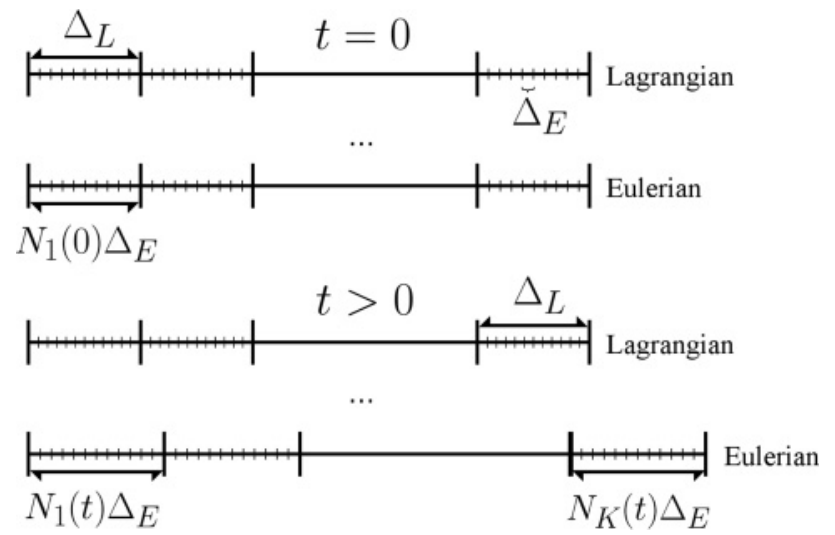

FIG. 7. Illustrating the connection between the Lagrangian description of the domain and the corresponding Eulerian description. See text for details. $i$ is then able to contain a number of further subdivisions $N_{i}$, which is identified as the number of Eulerian boxes in Lagrangian box $i$. As the domain evolves with stochastic fluctuations in the spatial variable the Lagrangian domain remains fixed by definition, with domain growth represented by alterations in the number of Eulerian boxes in each Lagrangian compartment. This has the immediate interpretation of each Eulerian box corresponding to a biological cell (or group of cells), with the number of cells varying in time within a given Lagrangian element and thus inducing domain growth.

The microscopic variable, $N_{i}$, corresponding to the number of Eulerian boxes in Lagrangian box $i$ is linked to a macroscopic variable, $n_{i}$, which defines the average ratio of the total size of the corresponding Eulerian subcompartments, $N_{i}(t) \Delta_{E}$, at a time $t$ to Lagrangian box size, $\Delta_{L}$, through

$$
n_{i}(t) \stackrel{\text { def }}{=} \frac{N_{i}(t) \Delta_{E}}{\Delta_{L}} \stackrel{\text { def }}{=} \frac{N_{i}(t)}{\theta} .
$$

Thus $\theta=\Delta_{L} / \Delta_{E}$ and is the scaling between the Lagrangian and Eulerian representations. Furthermore, for homogeneous initial conditions for the domain, which are assumed below, we have

$$
N_{i}(0) \Delta_{E}=\Delta_{L}
$$

and thus $n_{i}(0)=1$ for all $i$. Similar to the Eulerian description in Sec. II A, the population in each Lagrangian compartment is labeled $U_{i}$, for $i=1, \ldots, K$, and is related to the macroscopic ratio $\phi_{i}$ and the stochastic variable $\eta_{i}$ through $\Omega$, the initial average population, as

$$
U_{i}=\Omega \phi_{i}+\sqrt{\Omega} \eta_{i}
$$

In order to write down the CME for this system we must consider the effect of growth in the Eulerian coordinate system and how it is translated to the Lagrangian domain. Since the first-order term of the weak-noise expansion is the deterministic equation, we are led to derive a stochastic analog of Eq. (69). This is done by creating transition rates that mirror the effect of the deterministic time dependence of the diffusion coefficient $d_{L} / n(t)^{2}$ [see Eq. (69)].

Approaching the CME through the formalism of van Kampen's linear noise expansion method [6], at order $\sqrt{\Omega}$ we obtain the equations

$$
\begin{aligned}
\frac{\partial \phi_{1}}{\partial t} & =\frac{d_{L}}{n^{2}}\left(\phi_{2}-\phi_{1}\right) \\
\frac{\partial \phi_{i}}{\partial t} & =\frac{d_{L}}{n^{2}}\left(\phi_{i+1}-2 \phi_{i}+\phi_{i-1}\right) \quad \text { for } \quad i=2, \ldots, K-1, \\
\frac{\partial \phi_{K}}{\partial t} & =\frac{d_{L}}{n^{2}}\left(\phi_{K-1}-\phi_{K}\right) .
\end{aligned}
$$

Defining $d_{L}=D / \Delta_{L}^{2}$, where $D$ is the macroscopic diffusivity, we can see that on taking $K \rightarrow \infty$ or, equivalently, $\Delta_{L} \rightarrow 0$, we retrieve the discretized diffusion PDE mapped from the growing Eulerian domain onto a stationary Lagrangian domain [see Eq. (69) in Sec. IV]. 


\section{SPECTRA OF DIFFUSION ON DETERMINISTICALLY GROWING DOMAINS}

By using the Lagrangian formulation of growth we are able to invoke results from Sec. III, with the identifications $\Delta_{E} \mapsto \Delta_{L}$ and $d_{E} \mapsto d_{L} / n(t)^{2}$. Thus we can analytically derive the spatial Fourier cosine transformation of diffusion with the generalization that the diffusion coefficient is now time-dependent,

$$
\begin{aligned}
\left\langle\widehat{\eta}_{k}\left(t_{1}\right) \widehat{\eta}_{k^{\prime}}\left(t_{2}\right)\right\rangle= & \exp \left[-\left(C(k) \int_{0}^{t_{1}} \frac{1}{n^{2}} d t^{\prime}\right)\right. \\
& \left.-\left(C\left(k^{\prime}\right) \int_{0}^{t_{2}} \frac{1}{n^{2}} d t^{\prime}\right)\right] \frac{\Delta_{L}^{2} K \phi}{2} \\
& \times\left[\exp \left(2 C(k) \int_{0}^{\min \left(t_{1}, t_{2}\right)} \frac{1}{n^{2}} d t^{\prime}\right)-1\right] \delta_{k+k^{\prime}, 0} .
\end{aligned}
$$

This expression could be used to calculate the full power spectrum by the temporal Fourier transform Eq. (26). However, the resulting double integral would have to be evaluated numerically. Since we are primarily interested in systems that tend to a temporal steady state but may have inhomogeneous spatial behavior, our main concern lies with the spatial Fourier cosine transform that produces a complete, closed solution.

By varying the growth law we are able to derive the spatial power spectra of exponential growth, $n=e^{r t}$,

$$
\begin{aligned}
P_{s}(k, t) \stackrel{\text { def }}{=} & \frac{\left\langle\left.\widehat{\eta}_{k}(t)\right|^{2}\right\rangle}{\Delta_{L}^{2} K \phi}=\frac{1}{2}\left[1-\exp \left(\frac{2 d_{L}}{r}[1-\exp (-2 r t)]\right.\right. \\
& \left.\left.\times\left[\cos \left(k \Delta_{L}\right)-1\right]\right)\right]
\end{aligned}
$$

$$
\lim _{t \rightarrow \infty} P_{s}(k, t)=\frac{1}{2}\left[1-\exp \left(\frac{2 d_{L}}{r}\left[\cos \left(k \Delta_{L}\right)-1\right]\right)\right]
$$

linear growth, $n=1+\operatorname{tr} / \theta$,

$$
\begin{aligned}
P_{S}(k, t)= & \frac{1}{2}\left(1-\exp \left\{\frac{4 d_{L} \theta}{r}\left[1-\left(1+\frac{r}{\theta} t\right)^{-1}\right]\right.\right. \\
& \left.\left.\times\left[\cos \left(k \Delta_{L}\right)-1\right]\right\}\right), \\
\lim _{t \rightarrow \infty} P_{s}(k, t)= & \frac{1}{2}\left[1-\exp \left(\frac{4 d_{L} \theta}{r}\left[\cos \left(k \Delta_{L}\right)-1\right]\right)\right] ;
\end{aligned}
$$

and no growth, $n=1$,

$$
\begin{gathered}
P_{s}(k, t)=\frac{1}{2}\left(1-\exp \left\{4 d_{L} t\left[\cos \left(k \Delta_{L}\right)-1\right]\right\}\right), \\
\lim _{t \rightarrow \infty} P_{s}(k, t)=\left\{\begin{array}{lll}
0 & \text { if } & k=0 \\
\frac{1}{2} & \text { if } & k \neq 0 .
\end{array}\right.
\end{gathered}
$$

From these expressions we note that by reducing the growth rate, $r$, we reduce the difference between the growth and static domain spectra, as expected.

From Fig. 8 we see that growth affects the active spatial frequencies of diffusion. In the static domain case, all nonzero wavelengths will eventually have the same strength, as can be seen from Eq. (82), thus no spatial frequency can dominate. However, with linear and exponential growth, the lower spatial frequencies will have greatly reduced power for all time. Thus we expect to see consistent spatial heterogeneity occurring where growth is relatively fast. Individual stochastic realizations of diffusion on domains undergoing deterministic linear growth with different rates of growth are shown in Fig. 9. The simulations show many transient inhomogeneous states but, by considering Fig. 9(e), we clearly see consistently high population values in certain Lagrangian boxes lasting approximately 200 or more simulated time units that are not seen in the static domain case. This is corroborated by Fig. 9(f), which shows that spatial frequencies $m=2,3$, and 4 are being excited for long periods of time.

This implies that for single realizations of the system certain wavelengths will be excited; however, these wavelengths are chosen randomly among all the possible excited frequencies since we expect an averaged ensemble to smooth out individual peaks. This idea is best seen when the system undergoes extremely fast growth or, equivalently, for large values of $r$. In Fig. 10(a) $r=100$ and we see that once a heterogeneous state is reached it lasts for all remaining simulation time. Thus, although for a single simulation, as in Fig. 10(a), definite wavelengths set in [see Fig. 10(b)], when an ensemble of solutions are averaged, as in Fig. 10(d), they produce a power spectrum that matches the analytical solution [Fig. 10(c)], as expected.

The reason behind this persistent heterogeneity is understandable; initially the domain is quite small, so diffusion is able to carry the particles between the Lagrangian boxes and, due to the stochasticity of the simulated diffusion process, it is

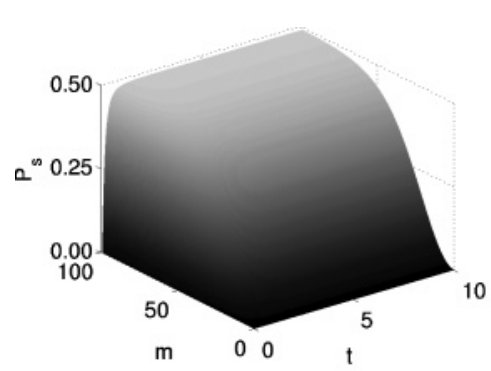

(a) Exponential growth.

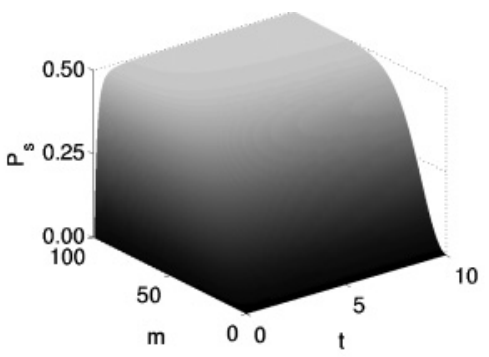

(b) Linear growth.

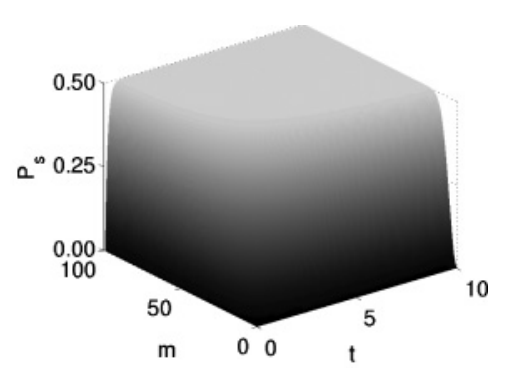

(c) No growth.

FIG. 8. Spatial power spectra of diffusion on exponentially and linearly growing domains, compared to that of a static domain. For exponential growth $r=1$, for linear growth $r / \theta=1$, and for all simulations $\Delta_{L}=1 / 100$ and $d_{L}=1$. Note that $k=m \pi / L$ and $L=1$. 


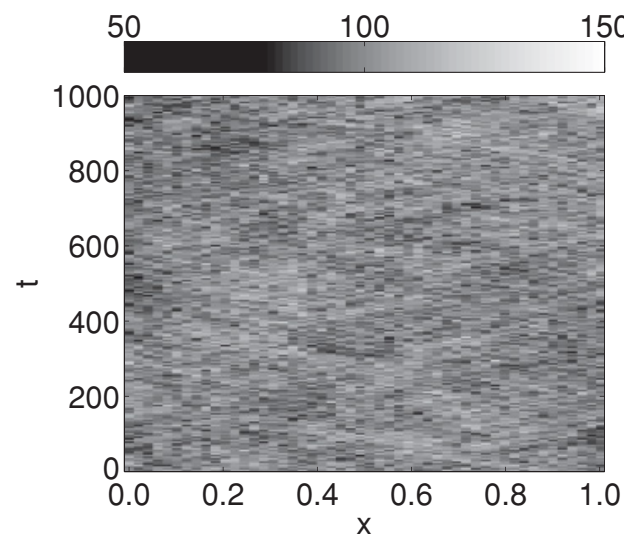

(a) $r=0.01$.

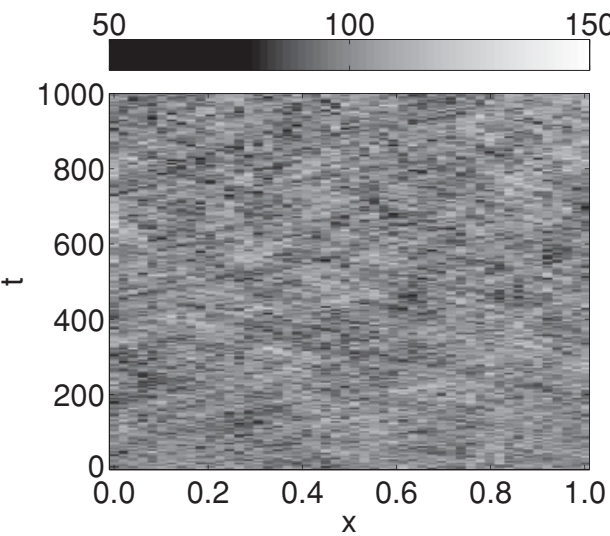

(c) $r=0.1$.

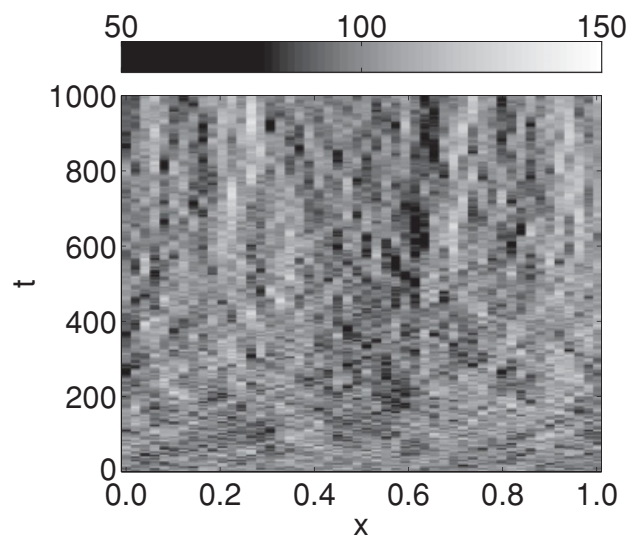

(e) $r=1$.

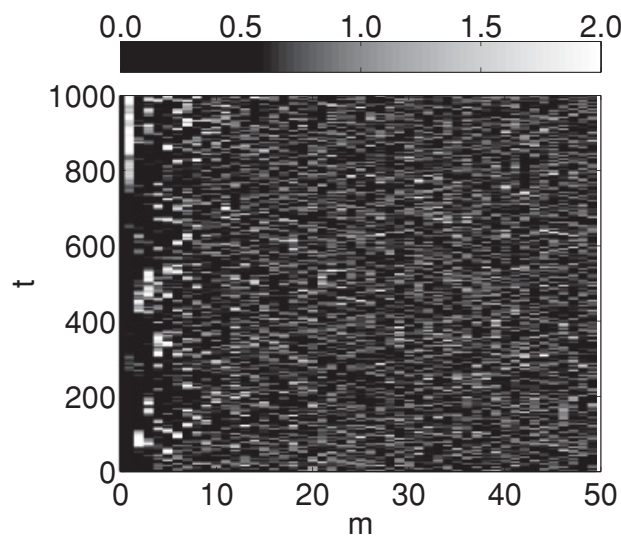

(b) $r=0.01$.

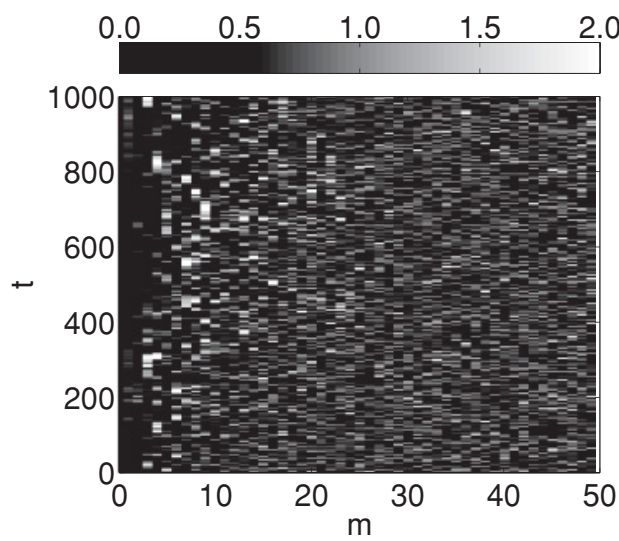

(d) $r=0.1$.

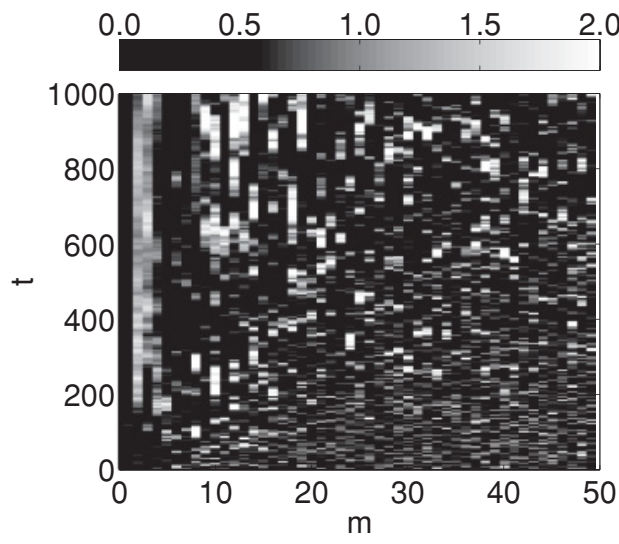

(f) $r=1$.

FIG. 9. Left column: single realizations of a diffusion process on a linearly growing domain (mapped onto the Lagrangian domain) for different rates of growth. Initially there were 100 Lagrangian boxes, each containing 100 simulated Eulerian boxes and each Lagrangian box contained 100 particles. The growth rate $r$ is noted beneath each figure; $\Delta_{L}=1 / 50$ and $d_{L}=1$. Right column: spatial Fourier cosine transformation of the realizations in left column.

highly likely that the system will produce an inhomogeneous state. If the system is not growing then this state is transient. However, in the case of fast growth, the system grows quicker than diffusion can transport particles. Thus each Lagrangian box effectively becomes decoupled from the rest of the system, causing any effects of spatial noise to be fixed for the rest of the simulation.

Specifically, we can capture this reasoning, in the linear growth case, by using the nondimensional parameter grouping $d_{L} \theta / r$. By considering Eq. (80) and by inspection of Fig. 9 we 


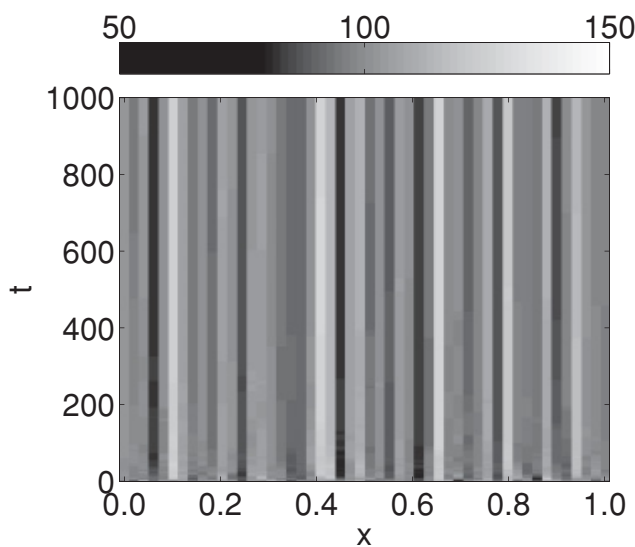

(a)

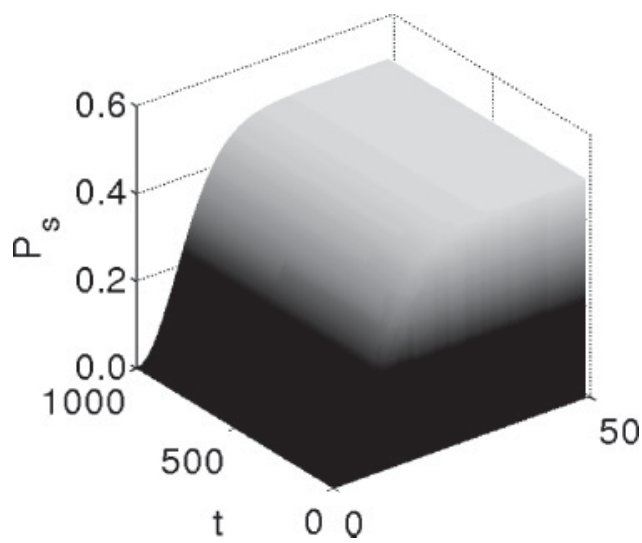

(c)

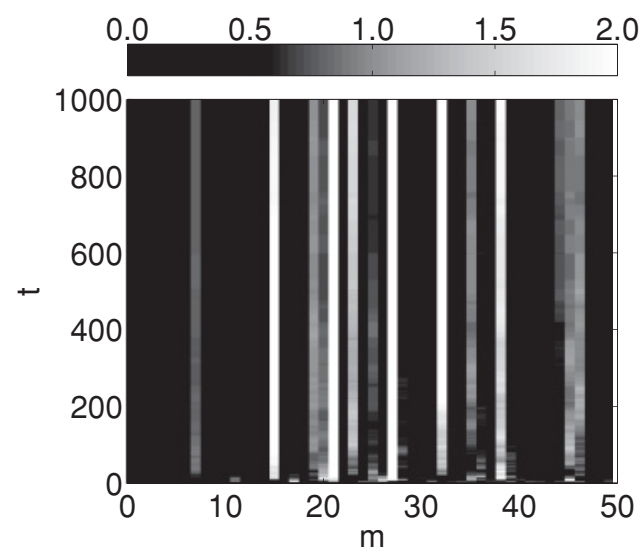

(b)

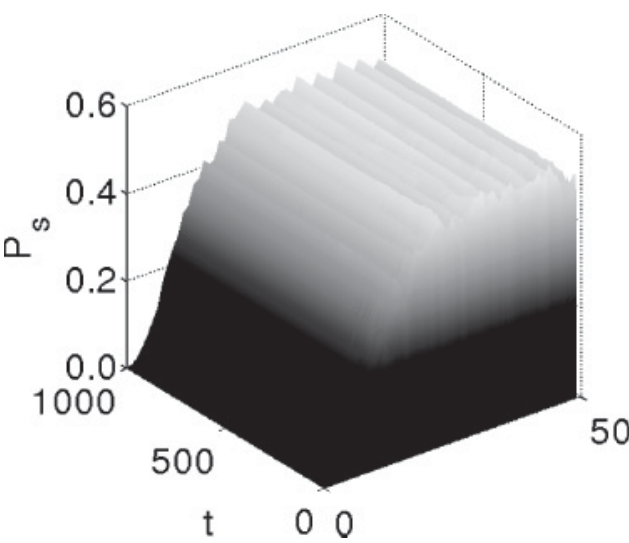

(d)

FIG. 10. (a) A single realization of a diffusion process on a fast linearly growing domain. The fixed pattern is indicative of the heterogeneities formed. (b) Power spectrum of the simulation in (a). Note that certain spatial frequencies are more excited than others. (c) Analytic power spectrum of diffusion on a linearly growing domain Eq. (80). (d) 1000 averaged power spectra derived from stochastic simulations of diffusion on a linearly growing domain. The parameters are $d_{L}=1, \Delta_{L}=1 / 50, r=100, k=m \pi / L, L=1$, and $\theta=100$.

see that the persistent peaks set in for $r>0.1$. Thus, for

$$
\frac{d_{L} \theta}{r}<1000
$$

we would expect nontransient heterogeneities to form. Otherwise diffusion will lead to homogeneity. Thus the stochastic structures depend on the balance of diffusion and growth, as expected. Due to this ratio being based on observation, Eq. (83) should only be used as an approximate value. Other growth forms will yield similar nondimensional parameter groupings.

To see that Eq. (83) is consistent with our physical intuition we consider approximate orders of realistic physical quantities. Using $D=10^{-6} \mathrm{~cm}^{2} / \mathrm{s}$ [33] and taking the view that each Lagrangian compartment $\Delta_{L}=1 \mathrm{~mm}$ is made up of a number of Eulerian subdivisions, which can be identified with the size of a cell, $\Delta_{E}=10 \mu \mathrm{m}$ [63], we obtain

$$
\frac{D \theta}{1000 \Delta_{L}^{2}}=\frac{10^{-10} \times 10^{2}}{10^{3} \times 10^{-6}}=10^{-5} / \mathrm{s}<r .
$$

Since $10^{5} \mathrm{~s}$ is approximately one day, this suggests that if a substance is diffusing in a one-dimensional tissue that is uniformly growing faster than one cell per day then we would expect inhomogeneities to form.

\section{CONCLUSION}

The intention of this paper has been to introduce two analytical methods for understanding stochastic systems. First, we wanted to discuss and clarify the recent use of Fourier-transform techniques on stochastic reaction-diffusion systems [59]. We illustrated the importance of understanding the dependence of the power spectra on the initial and final values and using the correct discrete expansion to compensate for the spatial boundary conditions. Using these ideas, we were able to produce a general formula for the temporal power spectrum of a one population system, which evolves under either steady- or non-steady-state dynamics Eq. (41). This result can easily be generalized to higher numbers of populations, although it may not have a closed form. These techniques were then further extended to the case of spatial systems and the application of the spatial Fourier cosine transform to a tridiagonal matrix, which introduced factors of $\cos \left(k \Delta_{x}\right)$, where $x=E$ or $L$ depending on the scale on which we are working. Through applying these methods 
to the case of simple diffusion on a stationary domain we derived a spatial power spectrum, which implied that all spatial frequencies were equally excited and thus we did not expect patterning. Finally, by considering the full spatiotemporal power spectrum (Fig. 5), we saw a singularity at $(k, \omega)=(0,0)$. This showed that the simulated data were temporally and spatially independent, as expected.

This brings us to the second aim of the paper: to introduce a spatial transform, which allows rigorous analytical treatment of stochastic effects on spatially extended, growing systems. It was seen that, due to the jumping transition rates depending on time, we were unable to obtain closed-form solutions when using the temporal Fourier transform. However, it was still possible to evaluate the integrals numerically and thus we were able to consider resonating temporal frequencies, if needed. Since the intended application of this theory was to consider stationary inhomogeneous spatial systems, it is appropriate to continue the development of the spatial transform. Furthermore, the benefit of this Lagrangian formulation is more important than simply being able to use the Fourier cosine transform. We are now able to treat the large-scale limits rigorously and thus link the mesoscopic and macroscopic dynamics directly, without the use of moment closure heuristics [30]. However, care should be taken to ensure that the initial domain discretization is fine enough to ensure that the final time compartment size will be able resolve any apparent spatial dynamics. Furthermore, due to the loss of information on the Eulerian domain that occurs when moving from a microscopic to a mesoscopic level of description (mentioned in the paper), we implicitly assume that, through the scaling of the diffusion coefficient, the rate of diffusion on the microscopic level is large enough to produce a statistically uniform distribution of particles over the Lagrangian box. In such extremes, where the diffusive length scale is larger than the system length scale required for well-mixed assumptions, the Lagrangian framework cannot be used.

Clearly, we have shown analytically and computationally that stochastic effects are critical when considering diffusing populations on a growing domain. The Fourier techniques have allowed us to highlight in generality that stochastic systems are able to exhibit much richer dynamics than if a system is treated purely as deterministic. Specifically, domain growth fundamentally changes the power spectrum of diffusion and, further, relatively quick growth supports the nucleation of states that are far removed from the deterministic mean-field steady state.

In this paper we have been interested in introducing analytical methods. In further studies [64] we extend the applications of these methods to encompass stochastically growing domains. These methods can then be expounded upon in the future and applied to different biological pattern formation systems [54,65-68] in order to gain insight into the effects of stochasticity and growing domains on pattern formation.

\section{ACKNOWLEDGMENTS}

T.E.W. would like to thank the Engineering and Physical Sciences Research Council for support. P.K.M. was partially supported by the Royal Society Wolfson Foundation.
[1] Mathematical Models for Biological Pattern Formation, edited by P. K. Maini and H. G. Othmer (Springer-Verlag, Berlin, 2001).

[2] P. K. Maini, K. J. Painter, and H. N. P. Chau, J. Chem. Soc. Faraday Trans. 93, 3601 (1997).

[3] J. D. Murray, Mathematical Biology I: An Introduction, 3rd ed., Vol. 1 (Springer-Verlag, Berlin, 2003).

[4] J. D. Murray, Mathematical Biology II: Spatial Models and Biomedical Applications, 3rd ed., Vol. 2 (Springer-Verlag, Berlin, 2003).

[5] P. Guptasarma, BioEssays 17, 987 (1995).

[6] N. G. van Kampen, Stochastic Processes in Physics and Chemistry, 3rd ed. (North-Holland, Amsterdam, 2007).

[7] T. Rudge and K. Burrage, Bull. Math. Biol. 70, 971 (2008).

[8] C. W. Gardiner, Handbook of Stochastic Methods (Springer, Berlin, 1985).

[9] C. Escudero, J. Stat. Mech. (2009) P07020.

[10] J. García-Ojalvo and J. M. Sancho, Phys. Rev. E 49, 2769 (1994).

[11] J. García-Ojalvo and J. M. Sancho, Phys. Rev. E 53, 5680 (1996).

[12] J. García-Ojalvo and J. M. Sancho, Noise in Spatially Extended Systems (Springer-Verlag, Berlin, 1999).

[13] F. Sagués, J. M. Sancho, and J. García-Ojalvo, Rev. Mod. Phys. 79, 829 (2007).

[14] S. Scarsoglio, F. Laio, P. D’Odorico, and L. Ridolfi, Math. Biosci. 229, 174 (2011).
[15] C. W. Gardiner, K. J. McNeil, D. F. Walls, and I. S. Matheson, J. Stat. Phys. 14, 307 (1976).

[16] A. J. McKane and T. J. Newman, Phys. Rev. Lett. 94, 218102 (2005).

[17] A. J. McKane, J. D. Nagy, T. J. Newman, and M. O. Stefanini, J. Stat. Phys. 128, 165 (2007).

[18] C. A. Lugo and A. J. McKane, Phys. Rev. E 78, 51911 (2008).

[19] T. Butler and N. Goldenfeld, Phys. Rev. E 80, 030902(R) (2009).

[20] D. T. Gillespie, J. Comput. Phys. 22, 403 (1976).

[21] D. T. Gillespie, J. Phys. Chem. 81, 2340 (1977).

[22] M. A. Gibson and J. Bruck, J. Phys. Chem. A 104, 1876 (2000).

[23] D. T. Gillespie, J. Chem. Phys. 115, 1716 (2001).

[24] S. S. Andrews and D. Bray, Phys. Biol. 1, 137 (2004).

[25] Y. Cao, D. T. Gillespie, and L. R. Petzold, J. Chem. Phys. 123, 054104 (2005).

[26] T. Tian and K. Burrage, J. Chem. Phys. 121, 10356 (2004).

[27] T. Leppänen, M. Karttunen, R. A. Barrio, and K. Kaski, Sup. Prog. Theor. Phys. 150, 367 (2003).

[28] F. Zheng-Ping, X. Xin-Hang, W. Hong-Li, and O. Qi, Chin. Phys. Lett. 25, 1220 (2008).

[29] A. Singh and J. P. Hespanha, in Stochastic Analysis of Gene Regulatory Networks Using Moment Closure, Proceedings of the 2007 American Control Conference (IEEE, Piscataway, NJ, 2007), pp. 1299-1304. 
[30] R. E. Baker, C. A. Yates, and R. Erban, Bull. Math. Biol. 72 719 (2010).

[31] A. L. Koch, in Advances in Microbial Ecology, edited by K. C. Marshall (Plenum Press, New York, 1990), pp. 37-70.

[32] J. R. Lawrence, G. M. Wolfaardt, and D. R. Korber, Appl. Environ. Microb. 60, 1166 (1994).

[33] R. Dillon, C. Gadgil, and H. G. Othmer, PNAS 100, 10152 (2003).

[34] E. J. Hinch, Perturbation Methods (Cambridge University Press, Cambridge, England, 1991).

[35] P. M. Kulesa, G. C. Cruywagen, S. R. Lubkin, M. W. J. Ferguson, and J. D. Murray, Acta. Biotheor. 44, 153 (1996).

[36] P. M. Kulesa, G. C. Cruywagen, S. R. Lubkin, P. K. Maini, J. Sneyd, M. W. J. Ferguson, and J. D. Murray, J. Theor. Biol. 180, 287 (1996).

[37] S. Kondo and R. Asai, Nature (London) 376, 765 (1995).

[38] E. J. Crampin, E. A. Gaffney, and P. K. Maini, Bull. Math. Biol. 61, 1093 (1999).

[39] J. D. Murray, E. A. Stanley, and D. L. Brown, Proc. R. Soc. London Ser. B 229, 111 (1986).

[40] A. Okubo, P. K. Maini, M. H. Williamson, and J. D. Murray, Proc. R. Soc. London Ser. B 238, 113 (1989).

[41] T. E. Woolley, R. E. Baker, E. A. Gaffney, and P. K. Maini (unpublished).

[42] J. Elf and M. Ehrenberg, Genome Res. 13, 2475 (2003).

[43] D. T. Gillespie, Annu. Rev. Phys. Chem. 58, 35 (2007).

[44] D. T. Gillespie, Physica A 188, 404 (1992).

[45] C. M. Guldberg and P. Waage, J. Prakt. Chem. 19, 69 (1879).

[46] G. Grimmett and D. Stirzaker, Probability and Random Processes (Oxford University Press, New York, 2001).

[47] T. Jahnke and W. Huisinga, J. Math. Biol. 54, 1 (2007).

[48] D. A. McQuarrie, J. Appl. Probab. 4, 413 (1967).

[49] H. Risken, The Fokker-Planck Equation: Methods of Solution and Applications (Springer, Berlin, 1989).
[50] R. Erban, S. J. Chapman, and P. K. Maini, e-print arXiv:0704.1908v2.

[51] K. W. Morton and D. F. Mayers, Numerical Solution of Partial Differential Equations: An Introduction (Cambridge University Press, Cambridge, England, 2005).

[52] A. Twomey, M.Sc. thesis, University of Oxford, 2007.

[53] D. T. Gillespie, Am. J. Phys. 64, 1246 (1996).

[54] T. Biancalani, D. Fanelli, and F. Di Patti, Phys. Rev. E 81, 046215 (2010).

[55] J. Andrew and A. J. McKane, J. Theor. Biol. 267, 85 (2010).

[56] T. Reichenbach, M. Mobilia, and E. Frey, Phys. Rev. E 74, 51907 (2006).

[57] W. L. Briggs and V. E. Henson, The DFT: An Owner's Manual for the Discrete Fourier Transform (SIAM, Philadelphia, 1995).

[58] We treat the singular case of linear growth separately.

[59] A. J. McKane and T. J. Newman, Phys. Rev. E 70, 041902 (2004).

[60] D. Alonso, A. J. McKane, and M. Pascual, J. R. Soc. Interface 4, 575 (2007).

[61] E. J. Crampin, PhD. thesis, University of Oxford, 2000.

[62] E. J. Crampin and P. K. Maini, Comments Theor. Biol. 6, 229 (2001).

[63] B. Alberts, D. Bray, J. Lewis, M. Raff, K. Roberts, and J. D. Watson, Molecular Biology of the Cell, 3rd ed. (Garland Science, New York, 1994).

[64] T. E. Woolley, R. E. Baker, E. A. Gaffney, and P. K. Maini (unpublished).

[65] R. A. Satnoianu and M. Menzinger, Phys. Rev. E 62, 113 (2000).

[66] W. Zeng, G. L. Thomas, and J. A. Glazier, Physica A 341, 482 (2004).

[67] R. A. Barrio, R. E. Baker, B. Vaughan Jr., K. Tribuzy, M. R. de Carvalho, R. Bassanezi, and P. K. Maini, Phys. Rev. E 79, 031908 (2009).

[68] T. E. Woolley, R. E. Baker, P. K. Maini, J. L. Aragón, and R. A. Barrio, Phys. Rev. E 82, 051929 (2010). 